\title{
Paisajes geomorfológicos relacionados con la clasificación de los suelos en planicies y terrazas de Tabasco, México
}

\section{Geomorphological landscapes related to the classification of soils in plains and terraces of Tabasco, Mexico}

\author{
Alfredo Isaac Brindis-Santos ${ }^{1}$, David Jesús Palma-López ${ }^{2, *}$, Joel Zavala-Cruz², Ena Edith Mata-Zayas ${ }^{3}$, \\ Yareni Itzel López-Bustamante ${ }^{3}$
}

${ }^{1}$ Facultad Maya de Estudios Agropecuarios. Universidad Autónoma de Chiapas. Carretera Catazajá-Palenque, km. 4. 29980, Catazajá, Chiapas, México.

${ }^{2}$ Colegio de Postgraduados, Campus Tabasco. Periférico Carlos A. Molina S/N, 86500, H. Cárdenas, Tabasco, México.

${ }^{3}$ División Académica de Ciencias Biológicas. Universidad Juárez Autónoma de Tabasco. Carretera Villahermosa-Cárdenas, km.0.5 $\mathrm{S} / \mathrm{N}$, entronque a Bosques de Saloya. 86150, Villahermosa, Tabasco, México.

* Autor para correspondencia: (D. Palma) dapalma@colpos.mx

\section{RESUMEN}

La cartografía y la clasificación de suelos a escala semidetallada contribuyen a la toma de decisiones sobre usos intensivos de tierras agrícolas a nivel de regiones pequeñas, municipios y tierras agrícolas comunales (ejidos). Este tipo de estudios son escasos en el trópico mexicano, en especial aquellos que relacionan los aspectos del paisaje con los suelos existentes en una región. El conocer estas relaciones permitirá a futuro realizar estudios de suelos más eficientes y económicos. El objetivo del trabajo fue zonificar los paisajes geomorfológicos y el relieve/modelado para clasificar unidades de suelos en las Planicies y Terrazas de Tacotalpa y Jalapa en Tabasco, México. Con base en el enfoque geopedológico se clasificaron y cartografiaron los paisajes geomorfológicos a escala 1:250000 y el relieve/modelado a escala 1:50000. A la misma escala se clasificaron y cartografiaron los grupos y unidades de suelo, con el apoyo de ortofotos, un modelo digital de elevación y verificación en campo. El 82\% de área de estudio corresponde a un área plana sobre sedimentos fluviales y palustres. El área restante corresponde a los paisajes de terrazas y lomeríos. Se cartografiaron catorce relieve/ modelados: cauce activo, cauce inactivo, dique natural alto y bajo, playa de meandro, llanura de inundación, cubeta de decantación, valle acumulativo, lomerío bajo ligeramente convexo a cóncavo, lomerío bajo ligeramente convexo, lomerío bajo a alto ligeramente convexo, lomerío alto moderadamente convexo, lomerío fuertemente convexo y valle erosivo. Se clasificaron seis grupos de suelo: Gleysols $65 \%$, Fluvisols 15\%, Lixisols 8\%, Luvisols 8\%, Alisols 4.02\% e Histosols 1\%, con 27 calificadores (primarios y secundarios). Este estudio a la escala 1:50000 permitió explicar la distribución geográfica de las unidades de suelo en las diferentes geoformas y relieves/modelados.

Palabras clave: Geopedología; SIG; Suelos tropicales y Clasificación de suelos.

\section{ABSTRACT}

The cartography and soil classification at a semi-detailed scale contributes to the decision making on intensive uses of agricultural land at the level of small regions, municipalities and communal agricultural land (hh). Studies of this type are scarce in the Mexican tropic, specifically those that relate landscape aspects with regional soils. Knowledge of these relations allowes more efficient and economic soil surveys. The objective of this work is to zone the geomorphological landscapes and relief/modeling to classify soil units in the plains and terraces of Tacotalpa and Falapa in Tabasco, Mexico. Based on the geopedological approach, geomorphological landscapes at 1:250000 scale and relief/modeling at 1:50000 scale were classified and mapped. At the same scales, soil groups and soil units were classified and mapped, with the support of orthophotos, a digital elevation model, and field verification. Eighty-two percent of the study area corresponds to a flat area on fluvial and marshy sediments. The remaining area corresponds to terraces and hills. Fourteen reliefs/models were mapped: active channel, inactive channel, high and low natural dam, meander beach, floodplain, decantation basin, cumulative valley, low hillock slightly convex to concave, low lomerio slightly convex, low to high lomerio slightly convex, moderately convex high hill, strongly convex hill, and erosive valley. Six soil groups were classified: Gleysols 65\%, Fluvisols 15\%, Lixisols 8\%, Luvisols 7\%, Alisols 4.02\%, and Histosols $1 \%$, with 27 qualifiers (primary and secondary). This study at the scale 1:50000 allows explaining the geographical distribution of the soil units in the different geoforms and relief/modeling.

Keywords: Geopedology; GIS; Tropical soils; Soil classification.
Universidad Nacional Autónoma de México.
Uares por pares es responsabilidad de

Este es un artículo Open Access bajo la licencia CC BY-NC- 


\section{Introducción}

La cartografía y la clasificación de suelos han sido áreas claves de investigación en las disciplinas de la ciencia del suelo (Hartemink y Bockheim, 2013; Brevik et al., 2016; Aksoy et al., 2017). La cartografia de suelos permite explicar la distribución espacial de las propiedades edáficas y su relación con el relieve (Buol et al., 1990; Bautista-Zuñiga et al., 2004; Miller y Schaetzl, 2014; Escribano et al., 2016), mientras que la clasificación de los suelos tiene como propósito organizar sus propiedades edáficas y dar una nomenclatura al suelo, con la intención de ubicar su localización y el área que ocupa en un territorio específico (Porta et al., 2013; IUSS Working Group, 2015). Estas dos disciplinas son útiles en la toma de decisiones sobre la agricultura, los recursos naturales, planes de desarrollo productivos y proyectos sociales (Zinck, 1989; Esfandiarpoor et al., 2009).

En el sureste mexicano, se han realizado estudios de clasificación de suelos a escala semidetallada y de reconocimiento (Bautista et al., 2015; Palma-López et al., 2017; Barbosa et al., 2005). En Tabasco la cartografía de suelos más conocida es de escala 1:250000 (Palma-López et al., 2007; Zavala-Cruz et al., 2016a). En contraste, la información a escala semidetallada y detallada es limitada, en algunos casos desactualizada y no es de fácil acceso (Martínez-Villegas, 2007; Zavala-Cruz et al., 2014), lo que dificulta la toma de decisiones sobre usos intensivos a nivel de regiones pequeñas, municipios y ejidos. Al respecto, los estudios de suelos a escalas mayores son costosos y requieren gran cantidad de tiempo para ser desarrollados por lo que son escasos en México, especialmente en el sureste del país. Como alternativa a las metodologías convencionales de levantamiento de suelos, la geopedología explica el complejo proceso de formación de suelos dentro de un marco controlado por la geomorfología (Bocco et al., 2010; Farshad, 2013), y se propone como un método de apoyo al levantamiento de suelo (Esfandiarpoor et al., 2010; Paladino et al., 2017). El enfoque geopedológico es una contribución geomorfológica y pedológica para conocer la distribución espacial y taxónomica de los suelos y, combinado con las técnicas cartográficas modernas, permite ahorrar tiempo y recursos al obtener la cartografía de geoformas contenedoras de los suelos, a escala detallada de una manera más rápida y precisa (Zinck, 2012).

En el sureste se han hecho algunos esfuerzos para utilizar este concepto en zonas limitadas que han permitido plantear que es posible optimizar el tiempo de ejecución de estudios a nivel de semidetalle, sin perder la precisión requerida en los estudios, en paisajes geomorfológicos de planicies, terrazas, lomeríos, valles y su diversidad de relieves (Bautista-Zuñiga et al., 2015; Zavala-Cruz et al., 2016a; López-Castañeda et al., 2017; Palma-López et al., 2017).

Por todo lo anterior, el objetivo de esta investigación fue zonificar la variabilidad de los suelos en Planicies y Terrazas de Tacotalpa y Jalapa, Tabasco (PTTJ), con base en el enfoque geopedológico (Zinck, 2012); clasificar los paisajes geomorfológicos a escala 1:250000, así como el relieve/modelado a escala 1:50000, y a esta escala determinar los grupos de suelos y sus calificadores (primarios y secundarios), partiendo de la hipótesis de que, al realizar la cartografía de suelo a escala semidetallada, se obtendrá una mayor variabilidad de suelos clasificados.

\section{Descripciones del área de trabajo, métodos y materiales estudiados}

\section{1. ÁREA DE TRABAJO Y MÉTODOS}

El área de estudio de PTTJ se ubica en la provincia fisográfica Llanura Costera del Golfo Sur, en los municipios de Jalapa y Tacotalpa, al sur del estado de Tabasco, México. Tiene una extensión de 138,143 ha, ubicada entre las coordenadas $17^{\circ} 31^{\prime} 57^{\prime \prime}$ y $17^{\circ} 47^{\prime} 44^{\prime \prime} \mathrm{N}$ y $92^{\circ} 42^{\prime} 55^{\prime \prime}$ y $92^{\circ}$ $54^{\prime} 22^{\prime \prime} \mathrm{O}$; limita al norte con los municipios de Macuspana y Centro, al sur y al este con el estado de Chiapas y al oeste con los municipios de Teapa y Centro (INEGI, 2016). El clima se caracteriza 
por ser cálido húmedo con abundantes lluvias en verano $\operatorname{Am}(\mathrm{f})$ en Jalapa y cálido húmedo con lluvias todo el año Af(m) en Tacotalpa, con precipitación media de 2500 a $4000 \mathrm{~mm}$ por año, y temperatura media de $26^{\circ} \mathrm{C}$. La hidrología está conformada por la parte media y baja de las cuencas de los ríos Grijalva, Teapa y Tacotalpa (Palma-López et al., 2007; INEGI, 2016).

La metodología consistió en cartografiar los paisajes geomorfológicos y relieve/modelados con el enfoque geopedológico para usarlos como base en la cartografia y clasificación de los suelos (Zinck, 2012).

\subsection{GARTOGRAFÍA DE PAISAJES GEOMORFOLÓGICOS Y RELIEVE/MODELADO}

La zonificación de paisajes geomorfológicos escala 1:250000 se mejoró a partir de regionalizaciones generadas por Ortiz-Pérez et al. (2005) y Zavala-Cruz et al. (2016a), y la nomenclatura de la cartografia del relieve/modelados a escala 1:50000 se adoptó de estudios geomorfológicos y geopedológicos de Palma-López et al. (2012) y Zavala-Cruz et al. (2012).

La cartografía geomorfológica se elaboró mediante la integración de las bases utilizando el Sistema de Información Geográfica (SIG) ARC GIS 9.3 (ESRI, 2004).

Los linderos de relieve/modelados se precisaron con el análisis de la:

a) Forma. Se identificaron características geométricas de la superficie terrestre (Castillo y Lugo-Hubp, 2011) y se agruparon zonas con espaciamiento similar de isolíneas expresadas en el modelo digital de elevación (MDE) a escala 1:50000 (INEGI, 201 1b);

b) Tono y textura, mediante fotointerpretación de ortofotos: E15d11f, E15d11e, E15d21a, E15d21b, E15d21c, E15d21d, E15d21el y E15d21f, a escala 1:20000 (INEGI, 2011a), lo cual ayudó a delimitar geoformas cóncavas con vegetación hidrófila;

c) Procesos geomorfológicos reportados en varios estudios (Ortiz-Pérez et al., 2005; Zavala et al., 2012; Palma-López et al., 2012; Bautista et al., 2015; Zavala-Cruz et al., 2016a; López-Castañeda et al., 2017); d) Altura sobre el nivel del mar (msnm) y pendiente $(\%)$. Este análisis se realizó con la generación del mapa ipsométrico y de pendientes (IMTA, 1989), basados en el MDE, por medio del programa ARC GIS 9.3; además, se consultaron mapas topográficos de INEGI y se verificaron en campo con GPS y brújula Brunton;

e) Tipo de roca y edad. Se determinó a partir de la carta geológico-minera (SGM, 2008).

\subsection{CARTOGRAFÍA DE SUELOS}

La cartografía de paisajes geomorfológicos y relieve/modelados se utilizó como base para definir los sitios de muestreo y barrenaciones de suelo en campo, así como para establecer relaciones de toposecuencia y biosecuencia con los suelos, de acuerdo con la accesibilidad y el tiempo requerido para el muestreo. Se seleccionaron dos transectos con dirección noreste a suroeste, tomando en cuenta la mayor variabilidad de relieve/modelados, pendientes, alturas, presencia de ecosistemas y agrosistemas; el primero se ubica en terrazas y planicies del noreste, en el municipio de Jalapa, y el segundo en terrazas y planicies del suroeste, en el municipio de Tacotalpa. La ubicación y orientación de los transectos fue con la finalidad de obtener la mayor diversidad de unidades de suelo en la zona de estudio (Zavala-Cruz et al., 2014). Se realizaron catorce perfiles de suelos, al menos uno por tipo de relieve/ modelado, distribuidos en ecosistemas y agrosistemas en PTTJ.

Adicionalmente, para disminuir el trabajo de campo se colectaron datos físicos y químicos de perfiles descritos en varios relieves/modelados de la zona (Zavala-Cruz et al., 2009; Zavala et al., 2016b). Los perfiles edafológicos fueron descritos de acuerdo con el manual de Cuanalo (1990). Se colectó un kg de suelo por cada horizonte en bolsas de polietileno, previamente etiquetadas y se secaron al aire y bajo sombra. Los parámetros físicos y químicos que se determinaron para cada horizonte fueron: $\mathrm{pH}$ medido con cloruro de potasio (1:2.5); $\mathrm{pH}$ determinado mediante potenciometría en agua (1:2); conductividad eléctrica (CE) con extracto de saturación de un suelo por medición 
electrolítica (cationes y aniones); el contenido de materia orgánica $(\mathrm{MO})$ por el método de Walkley y Black; nitrógeno total $(\mathrm{N})$ mediante Semi-micro Kjeldahl; fósforo extraíble $(\mathrm{P})$ por el procesamiento de P-Olsen; bases intercambiables $\left(\mathrm{K}+, \mathrm{Ca}^{2}{ }^{2}, \mathrm{Mg}+\right.$ y $\mathrm{Mg}^{2}$ ) y capacidad de intercambio catiónico $(\mathrm{CIC})$ mediante extracción con acetato de amonio $1 \mathrm{~N}, \mathrm{pH}$ 7.0 y cuantificación por absorción atómica; textura por el método de hidrómetro de Bouyoucos, con base en los criterios de la Norma Oficial Mexicana (NOM-021-REGNAT, 2000).

Para los horizontes de suelos orgánicos, la materia orgánica se cuantificó por el método de incineración (Soil Survey Staff, 2014). La clasificación del grupo de suelo y calificadores se llevó a cabo siguiendo la Base Referencial Mundial de Recurso Suelo (IUSS Working Group, 2015).

La verificación de algunos linderos de las unidades de suelo se hizo mediante 200 barrenaciones, con barrena tipo holandesa, hasta $120 \mathrm{~cm}$ de profundidad, y posteriormente se comparó con las características edafológicas (profundidad, color, textura) de los perfiles descritos y clasificados. Se elaboró la cartografia de unidades de suelos a escala 1:50000, por medio de SIG utilizando el programa ARG GIS 9.3.

\section{Resultados}

\subsection{PAISAJES GEOMORFOLÓGICOS Y RELIEVE/ MODELADO}

La zona de estudio PTTJ tiene tres paisajes geomorfológicos: planicie fluvial activa (PFA), terraza costera detrítica (TCD) y lomerío suave a inclinado volcánico (LSIV) (Tabla 1; Figura 1). El paisaje dominante es la PFA, con $82 \%$ de la superficie total, que es un área plana que ocupa la posición topográfica más baja, sobre sedimentos fluviales y palustres del Cuaternario Holoceno. El 18\% restante de la región corresponde a los paisajes de terrazas y lomeríos, dominados por procesos de erosión e intemperización, sobre rocas sedimentarias detríticas y brecha andesítica de edad Terciario Paleoceno al Cuaternario Pleistoceno, con alturas de 7 a $70 \mathrm{msnm}$ (Zavala-Cruz et al., 2016a).
En la PFA, los relieve/modelados son originados por acumulación de materiales aluviales gruesos a finos, de pendiente plana a concava, menores a $2 \%$ y altura de 8 a 27 msnm. En sentido perpendicular a los cauces de los ríos se desarrollan los relieve/ modelados:

a) Cauce activo, caracterizado por el patrón meándrico, abundancia de cauces inactivos y meandros estrangulados;

b) Dique natural alto y dique natural bajo, que se ubican a ambos lados de los cauces y actúan como barreras naturales contenedoras de los cauces; se forman por la mayor acumulación de sedimentos medios a finos durante los desbordamientos extraordinarios de los ríos; ocupan la posición topográfica más alta y su forma ligeramente convexa favorece el buen drenaje superficial; sobresalen en el río Tacotalpa y solo se inundan unos días al año en la época de lluvias;

c) Playa de meandro, que se localiza en la curva interna de los meandros del río Tacotalpa, entre el cauce y los diques naturales, se origina por migraciones de los meandros; tiene sedimentos limosos, pendientes suaves y puede inundarse unos días al año;

d) Llanura de inundación, que es la geoforma más extensa del área de estudio, varía de plana a ligeramente cóncava; los diques naturales la aíslan parcialmente de los cauces pero tiene conexión con los ríos a través de arroyos; prevalecen los sedimentos finos y medios; se inunda varios meses en la época de lluvias, por inundaciones fluviales y pluviales;

e) Cubeta de decantación, geoforma cóncava que ocupa la parte central de las llanuras de inundación en las zonas topográficas más bajas; su forma y las pendientes casi planas contribuyen a las inundaciones anuales en periodos mayores a seis meses; los sedimentos son arcillosos y orgánicos. En áreas próximas a las terrazas del sur existen pequeñas superficies de lomeríos bajos a altos ligeramente convexos, los cuales se encuentran en proceso de intemperización y tienen drenaje superficial moderado (Figura 2 y Tabla 1). 
El paisaje geomorfólogico de TCD está conformado por relieves planos a fuertemente convexos, con alturas de 7 a 46 msnm y pendientes de 2 a $30 \%$, con basamentos de rocas areniscas-lutitas en el noreste y lutitas-areniscas en el sureste, en su mayoría con buen drenaje superficial. Ha sido modelado por procesos de intemperización, erosión y acumulación. De la zona baja a la alta se presentan los relieve/modelados:

a) Lomerío bajo ligeramente convexo a cóncavo, que se caracteriza por ser una extensión de terreno con problemas de drenaje, dominado por los procesos de acumulación e intemperización; presenta la posición topográfica más baja y las pendientes más suaves; b) Lomerío bajo ligeramente convexo, cuya pendiente y altura aumentan respecto al lomerío anterior, además del proceso de intemperización inicia la erosión;

En forma ascendente se encuentran:

c) Lomerío bajo a alto ligeramente convexo,

d) Lomerío alto moderadamente convexo y

e) Lomerío alto fuertemente convexo; en estos lomeríos dominan los procesos de intemperización y erosión, con incremento de la altura y las pendientes son de moderadas a fuertes. También se presenta f) Valle acumulativo, que es un relieve plano (pendiente menor a $2 \%$ ), estrecho y alargado, situado entre los lomeríos, en transición con la PFA; acumula sedimentos fluviodeluviales aportados por

Tabla 1. Relieve/modelado y variabilidad de las unidades de suelos en el sur de Tabasco, México.

\begin{tabular}{|c|c|c|c|c|c|c|c|c|}
\hline \multirow{2}{*}{ 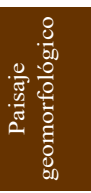 } & \multirow[b]{2}{*}{ Relieve/modelado } & \multirow[b]{2}{*}{ Unidades de suelo } & \multicolumn{4}{|c|}{ Datos morfométricos } & \multicolumn{2}{|c|}{ Superficie } \\
\hline & & & 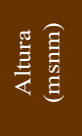 & 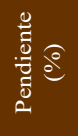 & Roca & $\begin{array}{l}0 \\
\vdots \\
0 \\
0 \\
0\end{array}$ & ha & $\%$ \\
\hline \multirow{9}{*}{ PFA } & Cauce activo & & $10-15$ & $<1$ & Sal & $\mathrm{A}, \mathrm{E}$ & 415 & 1.0 \\
\hline & Cauce inactivo & GL-st.fv.eu-ce.hu & $10-17$ & $<1$ & Sal & A & 797 & 2.0 \\
\hline & Dique natural alto & FL- fve.gl.st.eu- lo & $21-27$ & $2-4$ & Sal & A & 542 & 1.3 \\
\hline & Dique natural bajo & FL- fve.gl.eu- hu.lo & $10-24$ & $2-4$ & Sal & A & 5091 & 12.5 \\
\hline & Playa de meandro & FL- fve.eu- lo & $10-23$ & $2-7$ & Sal & $\mathrm{A}, \mathrm{E}$ & 991 & 2.4 \\
\hline & Llanura de inundación & GL- st.eu- lo.oh & $8-25$ & $0-2$ & Sal & A & 20417 & 50.0 \\
\hline & Cubeta de decantación & GL-hi.oy.eu- cen.lop.hu & $7-21$ & $0-2$ & $\mathrm{Sp}$ & A & 5197 & 12.7 \\
\hline & Lomerío bajo a alto ligeramente convexo & AL-fr-ct.ce.oh.pn & $15-30$ & $7-30$ & $\mathrm{Lu}-\mathrm{Ar}$ & $\mathrm{A}, \mathrm{I}$ & 70 & 0.2 \\
\hline & Lomerío bajo ligeramente convexo & AL-ab-cu.df.cen.lop.jd & $16-23$ & $7-15$ & Bva & $\mathrm{A}, \mathrm{I}$ & 74 & 0.2 \\
\hline \multirow{6}{*}{ TCD } & Valle acumulativo & GL- fl.eu- ce.hu & $7-15$ & $0-2$ & $\mathrm{Ar}-\mathrm{Lu}, \mathrm{Lu}-\mathrm{Ar}$ & A & 561 & 1.4 \\
\hline & Lomerío bajo ligeramente convexo a cóncavo & LV-gl- ct.hu-je.lo.qv & $10-13$ & $2-4$ & $\mathrm{Ar}-\mathrm{Lu}$ & A,I, & 1488 & 3.6 \\
\hline & Lomerío bajo ligeramente convexo & LX-ab.fr-ct.df.cen.lop.hu.je.pn & $13-17$ & $4-7$ & $\mathrm{Ar}-\mathrm{Lu}$ & A,I,E & 1557 & 3.8 \\
\hline & Lomerío bajo a alto ligeramente convexo & AL-fr-ct.ce.oh.pn & $15-30$ & $7-30$ & $\mathrm{Lu}-\mathrm{Ar}$ & $\mathrm{I}, \mathrm{E}$ & 426 & 1.0 \\
\hline & Lomerío alto moderadamente convexo & LV-ha-cu.cen.lop.je.oh & $17-34$ & $7-15$ & $\mathrm{Ar}-\mathrm{Lu}$ & $\mathrm{I}, \mathrm{E}$ & 908 & 2.2 \\
\hline & Lomerío alto fuertemente convexo & LV-ha-cu.cen.lop.je.oh & $35-46$ & $15-30$ & $\mathrm{Ar}-\mathrm{Lu}$ & $\mathrm{I}, \mathrm{E}$ & 214 & 0.5 \\
\hline \multirow{4}{*}{ LSIV } & Valle acumulativo & HS-hm.dr.rh.jd-mi & $20-25$ & $0-2$ & Bva & A & 260 & 0.6 \\
\hline & Valle erosivo & LX-ab.cr-ct.df.cen.lop.je.pn & $21-36$ & $15-30$ & Bva & $\mathrm{I}, \mathrm{E}$ & 9 & 0 \\
\hline & Lomerío bajo ligeramente convexo & AL-ab-cu.df.cen.lop & $16-23$ & $7-15$ & Bva & $\mathrm{I}, \mathrm{E}$ & 99 & 0.2 \\
\hline & Lomerío alto fuertemente convexo & LX-ab.cr-ct.df.cen.lop.je.pn & $24-70$ & $15-30$ & Bva & I,E & 1558 & 3.8 \\
\hline ea urbana & & & & & & & 149 & 0.4 \\
\hline
\end{tabular}

Paisaje geomorfológico: PFA/ Planicie fluvial activa, TCD/ Terraza costera detrítica, LSIV/ Lomerío suave a inclinado volcánico. Unidades de suelo: GL-st.fl.eu-ce.hu/Eutric Fluvic Stagnic Gleysol (Clayic, Humic), FL.fve.gl.st.eu-lo/ Eutric Stagnic Gleyic Pantofluvic Fluvisol (Loamic), FL-fve.gl.eu-hu.lo/ Eutric Gleyic Pantofluvic Fluvisol (Humic, Loamic), FLfve.eu-lo/ Eutric Pantofluvic Fluvisol (Loamic), GL-st.eu-lo.oh/Eutric Stagnic Gleysol (Loamic, Ochric), GL-hi.oy.eu-cen.lop.hu/ Eutric Oxigleyic Histic Gleysol (Endoclayic, Epiloamic, Humic), GL-fv.eu-ce.hu/Eutric Fluvic Gleysol (Clayic, Humic) LV-gl-ct.hu.je.lo.qv/ Gleyic Luvisol (Cutanic, Humic, Hypereutric, Loamic, Protovertic), LX-ab.frct.df.cen.lop.hu.je.pn/ Ferric Abruptic Lixisol (Cutanic, Differentic, Endoclayic, Epiloamic, Humic, Hypereutric, Profondic) AL-fe-ct.ce.oh.pn/ Ferric Alisol (Cutanic, Clayic, Ochric, Profondic), LV-ha-cu.cen.lop.je.oh/ Haplic Luvisol (Cutanic, Endoclayic, Epiloamic, Hipereutric, Ochric), HS-hm.dr.rh.jd-mi/Hyperdystric Rheic Drainic Hemic Histosol (Mineralic), LX-ap.cr-ct.df.cen.lop.je.pn/ Chromic Abruptic Lixisol (Cutanic, Differentic, Endoclayic, Epiloamic, Hipereutric, Profondic), AL-ab-cu.df.cen.lop.jd/ Abruptic Alisol (Cutanic, Differentic, Endoclayic, Epiloamic, Hyperdistric).Roca: Sal/ Sedimentos aluviales, Sp/ Sedimentos palustres, Lu/ Lutita, Ar Arenisca, Bva/ Brecha volcánica andesítica. Proceso Geomorfológico: A Acumulación, I Intemperización, E Erosión. 


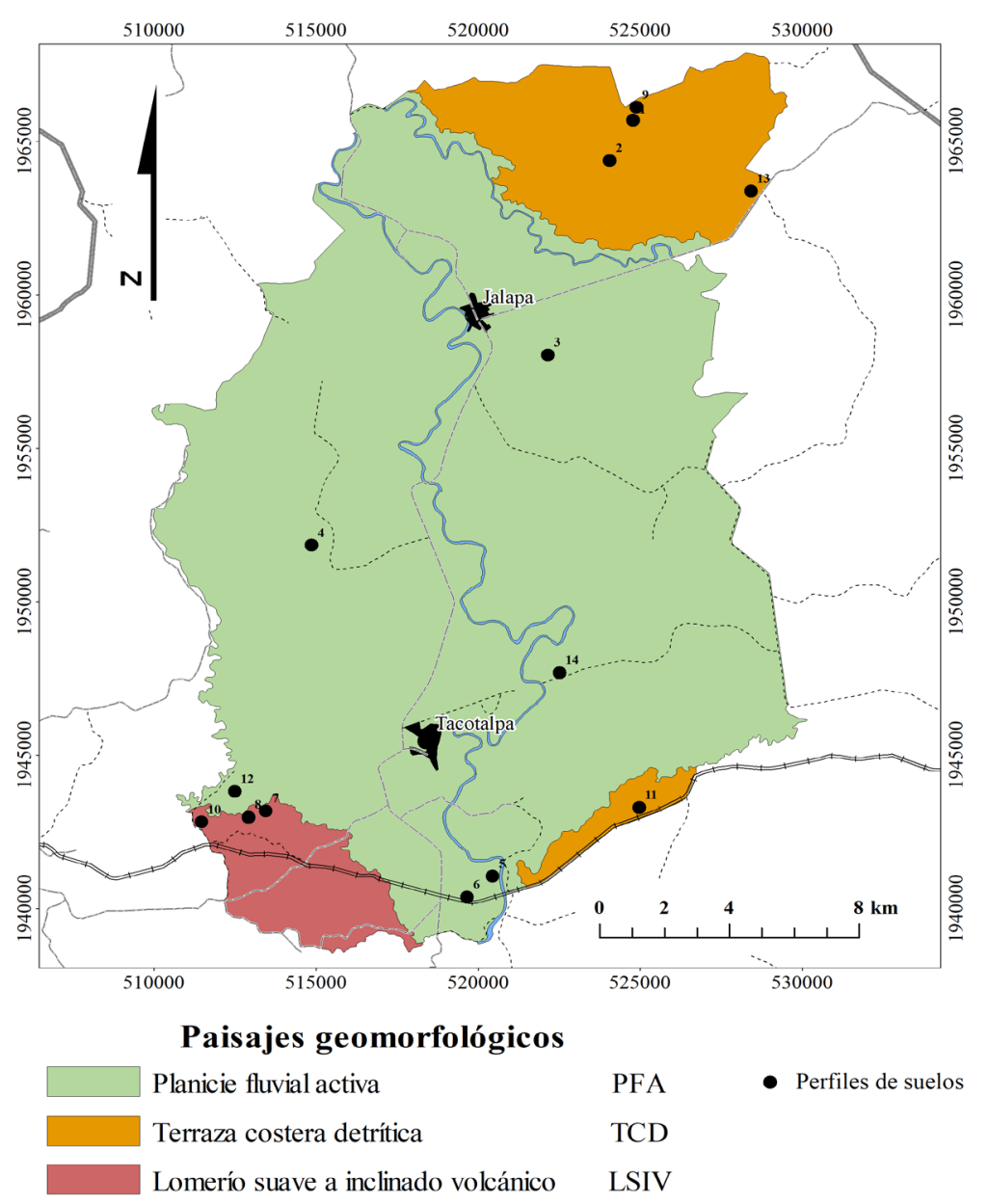

Figura 1 Paisajes geomorfológicos de la zona Sierra de Tabasco, México.

arroyos que drenan las terrazas altas; se inunda intermitentemente en la época de lluvias. La mayor área corresponde a lomeríos bajos ligeramente convexos, derivados de procesos de intemperización (Figura 2 y Tabla 1).

En el paisaje de LSIV, los relieves son de forma concava-convexa, sobre materiales de brecha volcánica andesítica, con una altura que varía entre los 20 y $70 \mathrm{msnm}$, con pendientes de 2 a $30 \%$ y, por lo tanto, son relieves susceptibles a procesos de intemperización y erosión. De la parte baja a la cima se encuentran los relieve/ modelados: a) Lomerío bajo ligeramente convexo; b) Lomerío alto fuertemente convexo; c) Valle acumulativo, y d) Valle erosivo. Dominan las superficies de lomerío alto fuertemente convexo con mayor efecto de los procesos erosivos (Figura 2 y Tabla 1).

\subsection{SUELOS EN LOS PAISAJES GEOMORFOLÓGICOS}

En los paisajes geomorfológicos de PTTJ se encuentran distribuidos seis grupos y 15 unidades de suelo sobre 14 relieve/modelados; cada relieve desarrolla una unidad diferenciada por uno o más calificadores, excepto los lomeríos altos de la TCD, que desarrollan la unidad de suelo Haplic Luvisols (Cutanic, Endoclayic, Epiloamic, Hypereutric, Ochric) LV-ha-cu. cen.lop.je.oh. La PFA tiene suelos poco desarrollados (perfiles con horizontes A/C), dominan los Gleysols (GL) y Fluvisols (FL). Los otros paisajes contienen 
suelos desarrollados (perfiles con horizontes A/B/C): en la TCD prevalecen los Luvisols (LV) y Lixisols (LX), y en el LSIV dominan los Lixisols (LX) (Figura 3 y Tabla 1).

\subsubsection{SUELOS DE LA PLANIGIE FLUVIAL ACTIVA (PFA)}

Los suelos asociados a los relieves de la PFA se formaron a partir de la acumulación de sedimentos aluviales y palustres, originando texturas francas, franco-arcillosas y arcillosas en el horizonte superficial. En los diques naturales y en las playas de meandro se desarrollan los Fluvisols (FL) (Tabla
1), presentando clases texturales de franca, franco-arenosa, franco-arcillosa o franco arcillo-limosa en el perfil (Figuras 3 y 4 ).

En los relieves de posición topográfica baja dominan los Gleysols con diversos calificadores (GL). Esta zona exhibe condiciones reductoras y un patrón de color gléyico debido al manto freático elevado y estancamiento de agua asociado a texturas medias y finas, lo que no favorece el drenaje interno pobre del perfil (Tabla 1). Los lomeríos aislados en la PFA desarrollan suelos típicos de la TCD (Figuras 3 y 4 ).

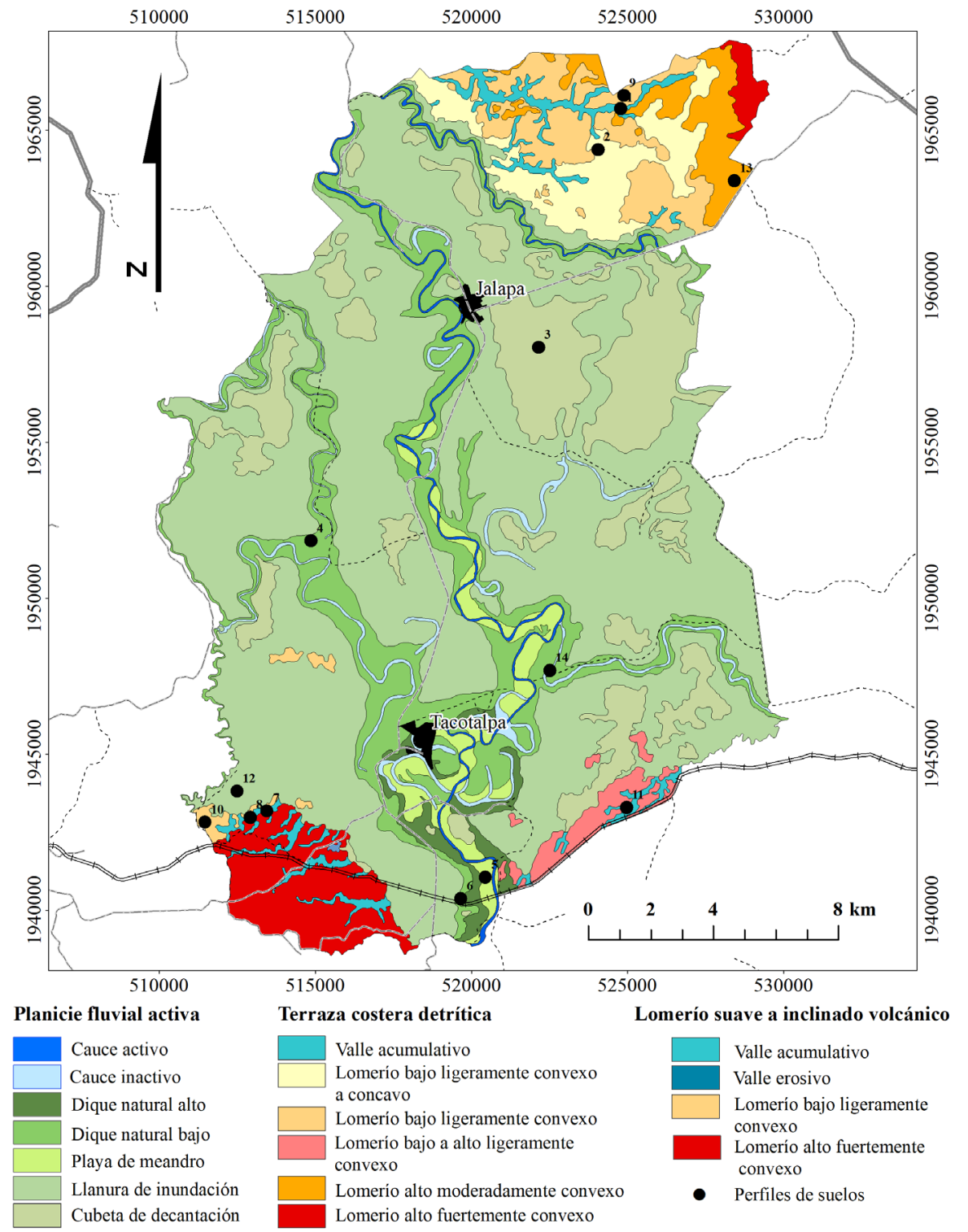




\subsubsection{SUELOS DE LA TERRAZA COSTERA DETRÍTICA (TCD)}

La TCD origina los suelos desarrollados de los grupos Luvisol (LV), Lixisol (LX) y Alisol (AL) sobre relieves de lomeríos. Todos los suelos presentan un horizonte Bt originado por la iluviación de la arcilla y diversos grados de acidez, esto último dependiendo de la baja saturación de bases de los LX y AL y de la moderada a alta saturación de bases en los LV (Tabla 1). Las diferencias de grupo y unidades se asocian al tipo de roca, ya que las dos terrazas difieren en su litología sedimentaria (areniscas-lutitas en el noreste y lutitas-areniscas en el sureste) y en su edad (del Terciario Paleoceno al Cuaternario Pleistoceno) (SGM, 2008). En los valles acumulativos, las pendientes casi planas reciben aportes de materiales y nutrimentos de los lomeríos, formando Gleysols; sobresale el proceso de gleyzación en los horizontes superficiales, debido a las inundaciones recurrentes y la saturación del perfil en la época de lluvias, además del contenido alto de materia orgánica (Figuras 3 y 4 ).

\subsubsection{SUELOS DEL LOMERÍO SUAVE A INCLINADO VOLCÁNICO (LSIV)}

Los suelos del paisaje LSIV, en el municipio de Tacotalpa, se desarrollaron en relieves de lomerío convexo, a partir de la roca brecha volcánica andesítica. La precipitación abundante todo el año de 3000 a $4000 \mathrm{~mm}$ y la alta temperatura de $26^{\circ} \mathrm{C}$ determinaron fuertes procesos de intemperización, erosión y lixiviación de las bases intercambiables. La interacción de estos factores originaron los AL y LX; ambos grupos de suelos tienen buen drenaje interno y texturas medias en los horizontes superficiales y arcillosas en los horizontes subsuperficiales debido al proceso de argilización (Tabla 1 y Figura 3). En los valles acumulativos más estrechos se forman suelos similares a la TCD, mientras que, en los valles con condiciones similares a las cubetas de decantación, se forman los suelos Histosols (HS). La presencia de este suelo orgánico en estos lomeríos resultó sorprendente dado el tipo de drenaje superficial existente en el entorno, pero se explica con la presencia de horizonte $\mathrm{C}$ mineral de textura gruesa en la profundidad (Figuras 3 y 4 ). Esta unidad representa el único suelo orgánico encontrado en el área de estudio.

\section{Discusión}

\subsection{AMBIENTES GEOMORFOLÓGICOS EN LA ZONA SIERRA DE TABASGO, MÉXICO}

Las Planicies y Terrazas de Tacotalpa y Jalapa se dividen en tres paisajes geomorfológicos: PFA, TCD y LSIV (Figura 1). Su fisiografía en general concuerda con regiones de planicies fluviales y terrazas detríticas reportadas como unidades ecogeográficas, paisajes geomorfológicos, unidades geomorfológicas y localidades a escala de reconocimiento (1:250000) reportadas por Ortiz-Pérez et al. (2005), Bollo-Manent y Hernández-Santana (2008), Solís-Castillo et al. (2014) y Zavala-Cruz et al. (2016a). Sin embargo, en este trabajo los linderos de los paisajes geomorfológicos de la PTTJ se mejoraron respecto a los estudios citados, al zonificar los relieves-modelados en el MDE y los ortofotomapas, con un criterio de área mínima cartografiable de 6 ha $\left(0.25 \mathrm{~cm}^{2}\right.$ de mapa $)$ a escala 1:50000. Posteriormente, al agrupar los patrones repetitivos de relieve/modelados de cada paisaje geomorfológico, resultaron unidades cartográficas con linderos más precisos a este nivel de geoforma.

En la PFA, la secuencia del relieve/modelados que ocupan mayor superficie, desde los cauces activos e inactivos hasta las zonas distales, es de diques naturales, llanura de inundación y cubetas de decantación. En el mismo sentido, varía el tipo de sedimentos aluviales de arenas a arcillas y el tiempo de inundación por periodos cortos hasta por más de seis meses al año; este arreglo de geoformas fluviales concuerda con la cartografía geomorfológica generada por Romo y Ortiz-Pérez (2001), Garnica-Peña y Alcántara-Ayala (2004), Solís-Castillo et al. (2014), Ramos-Reyes et al. (2016) 


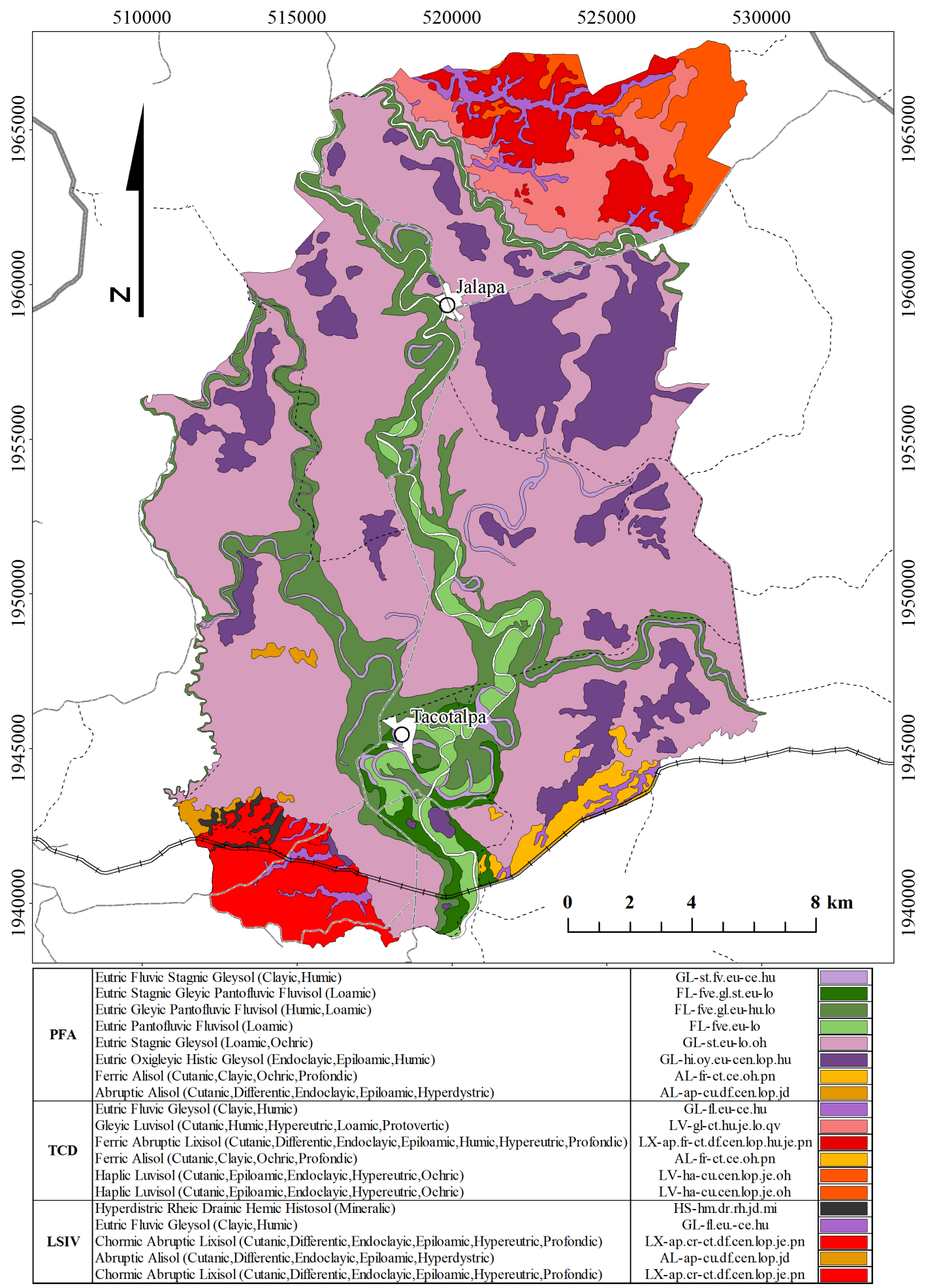


y Zavala-Cruz et al. (2016b) para diversas planicies fluviales. Se destaca el espacio ocupado $(6.6 \%$ de la PFA) por los relieves de cauces activos e inactivos, meandros estrangulados y playas de meandro, en el río Tacotalpa, que son evidencia de la intensa dinámica fluvial, caracterizada por migraciones laterales en las avenidas extraordinarias durante el Cuaternario Holoceno (Ortiz-Pérez et al., 2005; Zavala-Cruz et al., 2016a y 2016b) y por desembocar en los principales sistemas deltaicos y estuarios del Golfo de México (Lanza-Espino et al., 2013). Estos relieve/modelados y la interacción de procesos exógenos, principalmente por los hidrometeorológicos, se han observado para las zonas de llanura aluvial en Nayarit (Romo y Ortiz-Pérez, 2001; García-Sancho et al., 2009; Bojórquez et al. 2007), en las planicies ribereñas en Australia (Kemp et al., 2017) y en otras zonas fluviales del mundo (Martín-Vide, 2015; Harmand et al., 2017). Como anomalías de la PFA, en zonas próximas a las terrazas y lomeríos situados al sur, se localizan islas de lomeríos bajos a altos ligeramente convexos con materiales similares a los de la TCD, coincidiendo con lo reportado por Zavala-Cruz et al. (2016b), las cuales no aparecen identificadas en los mapas geomorfológicos a escala 1:250000 de Ortiz-Perez et al. (2005) y Zavala-Cruz et al. (2016a).

En la TCD se zonificó una secuencia de relieves con morfometría distinta. De la base a la cima se identificaron dos grupos. El primero corresponde a relieves negativos como el valle acumulativo y el lomerío bajo ligeramente inclinado a cóncavo. Ambos son casi planos, ocupan la posición topográfica más baja de la TCD, se ubican en transición con la PFA, y son receptores de flujos de agua y sedimentos aportados por los lomeríos convexos; en este contexto el valle sufre inundaciones estacionales. El segundo grupo corresponde a relieves positivos de bajos a altos de ligera a fuertemente convexos. Estos coinciden con laderas y cimas de pendiente suave a inclinada, bien drenadas, sujetas a intemperización y erosión, y por lo tanto transfieren materiales a los relieves negativos; los procesos endógenos y exógenos y tipo de roca coinciden con las características de las terrazas costeras de sedimentos detríticos zonificados por Zavala-Cruz et al. (2016b) y Ramos-Reyes et al. (2016). En otras terrazas costeras del sureste de México se reportan relieves similares clasificados como valle, terraza plana o inferior, terraza meso-mesiforme y terraza cumbral o interfluvio (Bollo-Manent y Hernández-Santana, 2008; Solís-Castillo et al., 2014).

Los relieves del paisaje de LSIV ocupan las mayores altitudes y pendientes del área de estudio. Sobresalen los lomeríos convexos modelados por procesos erosivos y denudativos, y los valles acumulativos, similares a geoformas de interfluvios, pendientes y valles cartografiados en llanuras onduladas del suroeste de Tabasco y noroeste de Chiapas (Bollo-Manent y Hernández-Santana, 2008).

\subsection{GEOPEDOLOGÍA EN LA SIERRA DE TABASCO, MÉXICIO}

Los paisajes geomorfológicos y relieve/modelados (Figuras 1 y 2) influyen en la variabilidad de los grupos y unidades de suelo en la zona de PTTJ (Figura 3 y Tabla 1). Los grupos de suelo asociados con la PFA tienen perfiles poco desarrollado con horizontes A-C y O-C. Los Gleysols (GL) son dominantes y se distinguen por su drenaje interno deficiente, asociado al manto freático elevado, que genera procesos de óxido-reducción. Estos suelos están asociados a relieves negativos en los paisajes de planicies fluviales (Ortiz-Pérez et al., 2005; Palma-López et al., 2007; Zavala-Cruz et al., 2016a y 2016b).

En la PFA todos los suelos tienen una saturación de bases intercambiables $\geq 50 \%$ entre el horizonte superficial hasta los $100 \mathrm{~cm}$ de profundidad, tienen buena fertilidad, debido al continuo aporte de material aluvial con altos contenidos de materia orgánica, y buena actividad biológica (Palma-López y Cisneros, 2000; Medina-Orozco et al., 2014; IUSS Working Group, 2015; Cabeza et al., 2017). Además, en los Fluvisols (FL) se presenta material flúvico dentro de más de un metro de profundidad debido a la gran cantidad de sedimentos aluviales 
a)

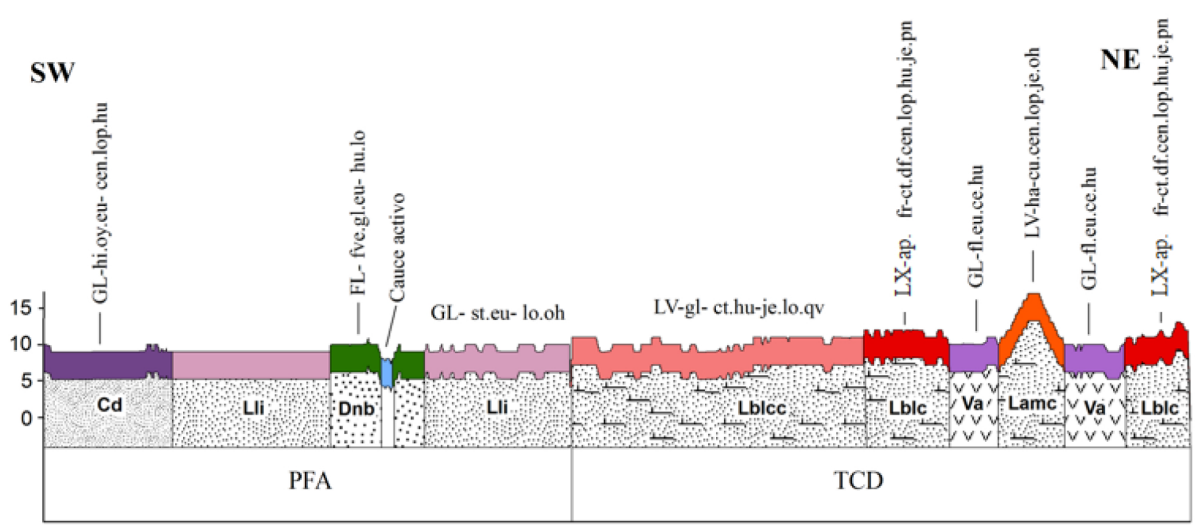

b)

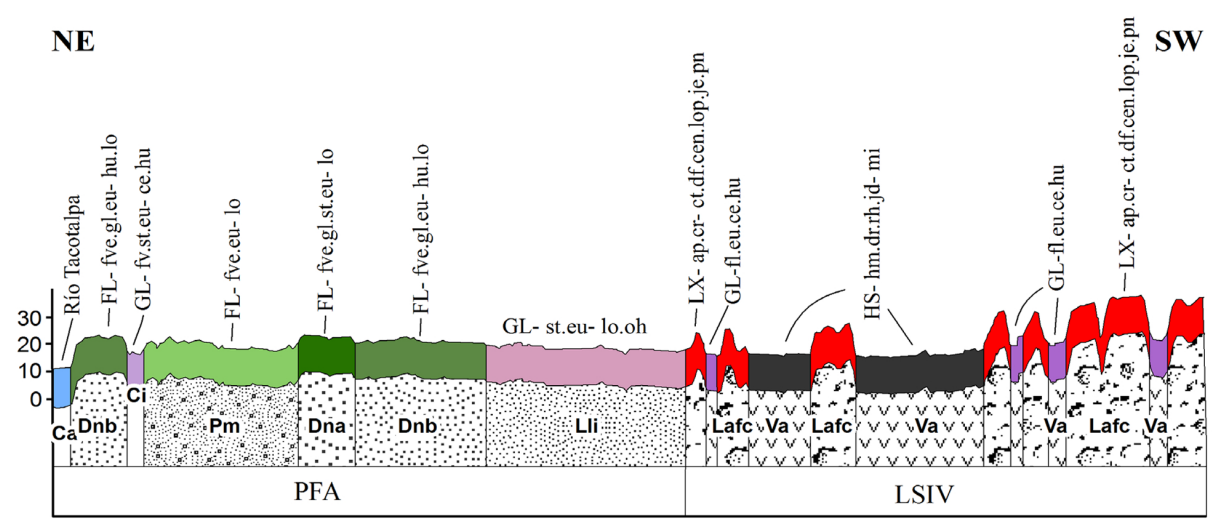

Figura 4 Transectos de relieve/suelo en la zona Sierra Tabasco, México. a) El Transecto de Suroeste a Noreste, presenta la secuencia de suelos poco desarrollados de la planicie fluvial y palustre GL/FL; la terraza tiene suelos desarrollados LX/LV. b) En el Transecto de Noreste a Suroeste, el paisaje PFA contiene los grupos FL/GL en diques naturales y llanuras de inundación; y en el paisaje LSIV, los lomeríos desarrollan el grupo LX, y los valles acumulativos el HS/GL.

que aportan los ríos y arroyos de la zona. Otro factor importante para estos suelos es la afectación por exceso de agua, que genera anaerobiosis en los horizontes subsuperficiales y procesos de óxido-reducción debidos al pobre drenaje interno (Figuras 3 y 4). Los Fluvisols son suelos muy productivos al ser ricos en nutrientes, por lo que suelen estar ocupados con cultivos semiperennes y cultivos básicos (Salgado-García et al., 2005; Palma-López et al., 2007; Bollo-Manent y Hernández-Santana, 2008; Zavala-Cruz et al., 2016b). Asimismo, los Fluvisols presentan diversidad en sus propiedades físicas, químicas y biológicas (Bedolla-Ochoa et al., 2017), provocada por el continuo aporte de sedimentos aluviales (Zavala-Cruz et al. 2016b; Ćirić et al., 2017). Dichos aportes originan que estos suelos presenten texturas Franco-limosas o Franco-arcillo-limosas en los horizontes superficiales, situación que los hacen ligeros y fáciles de trabajar. En los GL sobresale el estancamiento del agua superficial, que en la zona es provocado por el tiempo en el cual el suelo se encuentra saturado con agua superficial estancada, sobre todo en suelos con textura arcillosa o que se ubican en pendientes cóncavas, lo que permite que ocurran condiciones reductoras y, como característica particular, la combinación de colores azulosos y grisáceos, dentro de los agregados, y colores rojizos o amarillentos en los poros y paredes de agregados, donde ambos procesos son indicadores de reducción-oxidación alternados debido a las épocas de lluvias y secas (Gómez et al., 2010; IUSS Working Group, 2015). 
La terraza costera detrítica (TCD), con geoformas de altitudes bajas y relieves ligeramente convexos a cóncavos, así como los terrenos altos con relieves de moderados a fuertemente convexos, se caracteriza por desarrollar en el horizonte subsuperficial un mayor contenido de arcilla, como resultado de procesos edafogenéticos ligados a la migración de arcilla, presencia de barnices o revestimientos de arcilla y formación del horizonte árgico. Estos suelos son más desarrollados, con horizontes A-B-C, y pertenecen a los Grupos Luvisol (LV), Lixisol (LX) y Alisol (AL). Al respecto, Zavala-Cruz et al. (2016a y 2016b) indican que, en el trópico húmedo, estos grupos se caracterizan por presentar horizontes árgicos y revestimientos de arcilla, $\mathrm{pH}$ ácido y drenaje de bueno a imperfecto. En los lomeríos con altitudes bajas a altas con relieve convexo es notable la segregación de Fe en los LX y AL, lo que generalmente se acompaña con una pobre agregación de las partículas del suelo en las áreas empobrecidas en Fe y la compactación del horizonte en las zonas enriquecidas en Fe (IUSS Working Group, 2015). En los suelos LV, LX y AL se tienen procesos pedogénicos similares que se evidencian por barnices de arcilla (revestimiento) en el horizonte árgico. Esto es originado por la migración de arcillas hacia los horizontes inferiores, más de 1\% de carbono orgánico del suelo en la fracción tierra fina como promedio ponderado hasta una profundidad de $50 \mathrm{~cm}$ (debido a la acumulación de materia orgánica en formas húmicas residuales características en los suelos ácidos) y, para el caso del LV, una saturación de bases $\geq$ $50 \%$ en todo el espesor entre 20 y $100 \mathrm{~cm}$ de la superficie del suelo.

Hay texturas diferenciales al presentarse texturas arcillosas entre los 50 y $100 \mathrm{~cm}$ de profundidad, así como texturas francas dentro de los primeros $50 \mathrm{~cm}$ de profundidad, propiedades que permiten diferenciar a estos suelos para su uso agropecuario, ya que se presenta compactación debido al incremento de arcilla en los horizontes inferiores. En cada relieve/modelado, la distribución de las unidades de suelo concuerda con la composición de la roca y con los procesos geológicos ocurridos en la región (que han sido modificados por otros factores de formación de suelos, principalmente el relieve y el clima, durante el tiempo en que ha operado el intemperismo). Estas condiciones son evidentes en las terrazas costeras del Golfo de México (Zavala-Cruz et al 2014; Salgado-García et al., 2015; Zavala-Cruz et al., 2016a). El suelo GL del valle acumulativo resultó similar a los GL de la PFA.

Los Lixisols (LX) son los suelos principales de los lomeríos convexos del paisaje LSIV. Al presentarse un clima tropical muy lluvioso sobre los materiales geológicos más antiguos del área de estudio, se ha provocado una fuerte erosión y lixiviación de materiales en los suelos (Palma-López et al., 2007).

Adicionalmente, la zona se asocia a extensiones de pastizales con exceso de pastoreo, lo que provoca en la capa superficial degradación, con baja estabilidad de los agregados (Salgado-García et al., 2015).

En los valles acumulativos similares a los de la TCD se presenta el grupo de suelo GL. Sin embargo, en los valles con condiciones similares a las cubetas de decantación, se forman los Histosols (HS). Este suelo orgánico en los lomeríos resulta inusual debido a que los valles acumulativos en la época actual se inundan por periodos cortos en la época de lluvias y acumulan sedimentos minerales predominantemente. Sin embargo, en el Cuaternario Holoceno estos valles funcionaron como zonas lacustres y palustres, y aunado a las abundantes precipitaciones de la zona, propiciaron la acumulación de materia orgánica y la formación de HS, por lo que podría tratarse de paleosuelos (Figuras 3 y 4). En estos suelos existe saturación de agua en el horizonte superficial donde domina el material orgánico (carbono orgánico del suelo $\geq 20 \%$ medianamente descompuesto, lo que provoca colores pardo rojizo oscuro y olivo muy oscuro en el material orgánico, lo que indica el ralentizamiento de la descomposición de la materia orgánica. El suelo tiene permeabilidad lenta, por lo que los productores requieren utilizar drenaje artificial para implementar los usos agropecuarios. 
Sin embargo, se encuentra saturado con agua freática dentro de los $100 \mathrm{~cm}$ de profundidad, por lo que la productividad del suelo es baja.

Dentro de los Histosols, los suelos (como éste) que presentan $\mathrm{pH}$ inferior a 5.5 en las capas orgánicas se consideran como pobres en nutrimentos. Lo anterior se debe principalmente a que la relación carbono/nitrógeno $(\mathrm{C} / \mathrm{N})$ en todos los horizontes es muy alta (> 25), lo que indica inmovilización orgánica de $\mathrm{N}$ y baja taza de mineralización neta (IUSS Working Group, 2015; NOM-021-RECNAT, 2000). Esta unidad de suelo no fue reportada en la cartografía de suelos a escala 1:250000, generada en el área de estudio por Ortiz-Pérez et al. (2005), Palma-López et al. (2007), Zavala-Cruz et al. (2011 y Zavala-Cruz et al. (2016a). Sin embargo, algunos calificadores han sido reportados en diversos estudios asociados con diferentes usos y ecosistemas por Moreno-Cáliz et al. (2004), Arnalds (2004), Covelo et al. (2008) y Dominguez-Domiguez et al. (2011).

\section{Conclusiones}

La zonificación de los paisajes geomorfológicos y de los relieve/modelados a escala semidetallada 1:50000, con enfoque geopedológico, permitió determinar la variabilidad de las unidades de suelo en la Planicies y Terrazas de Tacotalpa y Jalapa en Tabasco, México. Se cartografiaron tres paisajes geomorfológicos, planicie fluvial activa, terraza costera detrítica y lomerío suave a inclincado volcánico. Dentro de ellos se diferenciaron 14 relieve/modelados.

El 82\% de la superficie corresponde al paisaje de planicie fluvial activa, caracterizado por presentar relieves dominantes de diques naturales, playas de meandro, llanuras de inundación y cubetas de decantación, con procesos de acumulacíon de sedimentos aluviales y palustres y pendientes menores al $2 \%$, por lo que estan expuestos a inundaciones recurrentes. Los suelos encontrados en estos relieves se muestran plenamente diferenciados: en los relieves planos a cóncavos se desarrollan los Gleysols, con estancamiento de agua durante largos periodos o bien, Gleysols que estuvieron saturados de agua, provocando colores reductimórficos. En los de posición ligeramente convexa el grupo dominante es el Fluvisol; en ellos predominan los depósitos recientes que originan las texturas franco-limosas a franco-arcillo-limosas, desde el horizonte superficial a los subyacentes, y presentan, además, estratificación profunda en el perfil de suelo.

En los paisajes de terrazas y lomeríos con basamentos de roca lutita-arenisca y brecha volcánica los suelos son más antiguos, ácidos y desarrollados. Predominan los Alisols, Luvisols y Lixisols; todos ellos presentan acumulación iluvial de arcilla que forma un horizonte árgico, con concentraciones residuales de óxidos de hierro y aluminio, así como una estructura subangular.

Además, algunos suelos contienen más del $1 \%$ de carbono orgánico hasta los $50 \mathrm{~cm}$ de suelo y tienen una alta saturación de bases. En los relieves de valles acumulativos, los grupos asociados son los Histosols y Gleysols, con una génesis asociada a las condiciones de anaerobiosis que trae como consecuencia procesos de gleyzación y acumulación de materia orgánica.

\section{Agradecimientos}

Al National Science Foundation Partnerships in International Research and Education (NSF), a través del proyecto "Sustentabilidad, Servicios Ecosistémicos y Desarrollo de la Bioenergía en las Américas" (PIRE) y al Consejo Nacional de Ciencia y Tecnología (CONACYT) por el financiamiento parcial de este trabajo.

\section{Referencias}

Aksoy, E., Louwagie, G., Gardi, C., Gregor, M., Schröder, C., Löhnertz, M., 2017, Assessing soil biodiversity potentials in Europe: Science of The Total Environment, 589, 236-249. https:// doi.org/10.1016/j.scitotenv.2017.02.173 
Arnalds, O., 2004, Volcanic soils of Iceland: Catena, 56(1-3), 3-20. https://doi. org/10.1016/j.catena.2003.10.002

Barbosa, O.A., Bertani, L.A., Fernandez, F.R., Mendoza, R.P., 2005, Análisis fisiográfico para el levantamiento pedológico semidetallado: Revista Brasileira de Engenharia Agrícola e Ambiental, 9(2), 191-198. https://doi. org/10.1590/s1415-43662005000200007

Bautista-Zuñiga, F., Estrada-Medina, H., JiménezOsornio, J.J.M., González-Iturbe, J.A., 2004, Relación entre el relieve y unidades de suelo en zonas cársticas de Yucatán: Terra Latinoamericana, 22, 243-254.

Bautista-Zuñiga, F., Frausto, O., Ihl, T., Aguilar, Y., 2015, Actualización del mapa de suelos del Estado de Yucatán, México: Enfoque geomorfopedológico y WRB: Ecosistemas y recursos agropecuarios, 2, 303-315.

Bedolla-Ochoa, C., Bautista, F., Ihl, T. \& Dubrovina, I. 2017, Diversidad de suelos y su distribución espacial. En: La biodiversidad en Michoacán. Estudio de Estado 2, vol. I. conabio, México, pp. 55-63.

Bocco, G., Mendoza, M.E., Priego, A., Burgos, A., 2010, La cartografía de sistemas naturales como base geográfica para la planeación territorial: México, Semarnat, 71 p.

Bojórquez, I., Hernández, A., García, D., Nájera, O., Flores, F., Madueño, A., Bugarín, R., 2007, Características de los suelos cambisoles y fluvisoles de la llanura costera norte del estado de Nayarit, México: Cultivos Tropicales, 28, 19-24.

Bollo-Manent, M., Hernández-Santana, J.R., 2008, Paisajes físico-geográficos del noroeste del estado de Chiapas, México: Investigaciones geográficas, 66, 7-24.

Buol, S.W., Hole, F.D., McCracken, R.J., 1990, Génesis y clasificación de suelos: México, Trillas, $417 \mathrm{p}$.

Brevik, E.C., Calzolari, C., Miller, B.A., Pereira, P., Kabala, C., Baumgarten, A., Jordán., 2016, Soil mapping, classification, and pedologic modeling: History and future directions:
Geoderma，264， 256-274. https://doi. org/10.1016/j.geoderma.2015.05.017

Cabeza, R.A., Myint, K., Steingrobe, B., Stritsis, C., Schulze,J., Claassen, N., 2017, Phosphorus fractions depletion in the rhizosphere of young and adult maize and oilseed rape plants: Journal of Soil Science and Plant Nutrition, 17(3), 824-838. https://doi. org/10.4067/s0718-95162017000300020

Castillo, M., Lugo-Hubp, J., 2011, Estado actual del conocimiento, clasificación y propuesta de inclusión del término Knickpoint en el léxico geológico-geomorfológico del español: Boletín de la Sociedad Geológica Mexicana, 63(2), 353-364. https://doi.org/10.18268/ bsgm2011v63n2a15

Ćirić, V.I., Drešković, N., Mihailović, D.T., Mimić, G., Arsenić, I., Đurđević, V., 2017, Which is the response of soils in the Vojvodina Region (Serbia) to climate change using regional climate simulations under the SRES-A1B?: Catena, 158, 171-183. https://doi.org/10.1016/j. catena.2017.06.024

Cuanalo de la, C.H., 1990, Manual de descripción de perfiles de suelo en el campo: Chapingo, Centro de Edafología, Colegio de Postgraduados, $40 \mathrm{p}$.

Covelo, E.F., Vega, F.A., Andrade, M.L., 2008, Sorption and desorption of Cd, $\mathrm{Cr}, \mathrm{Cu}, \mathrm{Ni}, \mathrm{Pb}$ and $\mathrm{Zn}$ by a Fibric: Journal of Hazardous Materials, 159(2)3, 342-347. https://doi.org/10.1016/j. jhazmat.2008.02.025

Domínguez-Domínguez, M., Zavala-Cruz, J., Martínez-Zurimendi, 2011, Manejo forestal de los manglares de Tabasco: Villahermosa, Tabasco, Colegio de postgraduados, $137 \mathrm{p}$.

Escribano, P., Oyonarte, C., Cabello, J., Zinck, J.A., 2016, Ecological land zonation using integrated geopedologic and vegetation information: Switzerland, Case study of the Cabo de GataNíjar Natural Park, Almería, Spain, in Zinck, J.A., Metternicht, G., Bocco, G., Del Valle, H.F., (eds.), Geopedology: Springer, 475-487. 
Esfandiarpoor, I.B., Salehi, M.H., Toomanian, N., Mohammadi, J., 2009, The effect of survey density on the results of geopedological approach in soil mapping: A case study in the Borujen region, Central Iran: Catena, 79(1), 18-26. https://doi.org/10.1016/j. catena.2009.05.003

Esfandiarpoor, I.B., Mohammadi, J., Salehi, M.H., Toomanian, N., Poch, R.M., 2010, Assessing geopedological soil mapping approach by statistical and geostatistical methods: A case study in the Borujen region, Central Iran: Catena, 82(1), 1-14. https:// doi.org/10.1016/j.catena.2010.03.006

Environmental System Research Institute (ESRI), 2004, ArcGis 9. What is ArcGis?: Redlands, Environmental System Research Institute, $119 \mathrm{p}$.

Farshad, A., 2013, Geopedology reports historical changes in climate and agroecology: a case study from Northwestern Iran: ECOPERSIA, 1, 145-158.

García-Sancho, A.G., Bojórquez-Serrano, J.I., Nájera-González, O., García-Paredes, J.D., Madueño-Molina, A., Flores-Vilchez, F., 2009, Regionalización ecológica de la llanura costera norte de Nayarit, México: Investigaciones geográficas, 69, 21-32.

Garnica-Peña, R.J., Alcántara-Ayala, I., 2004, Riesgos por inundación asociados a eventos de precipitación extraordinaria en el curso bajo del río Tecolutla, Veracruz: Investigaciones geográficas, 55, 23-45.

Gómez, I.A., Hernández, A., Ascanio, M.O., Sánchez, M.E., Durán, J.L., Benítez, L., Villegas, R., Ponce de León, D., López, M., 2010, Grupos de suelos y su distribución en las áreas del ingenio central Motzorongo, Veracruz, México: Cultivos Tropicales, 31, 32-36.

Harmand, D., Adamson, K., Rixhon, G., Jaillet, S., Losson, B., Devos, A., Hez, G., Calvet, M., Audra, P., 2017, Relationships between fluvial evolution and karstification related to climatic, tectonic and eustactic forcing in temperate regions: Quaternary Science Reviews, 166, 38-56. https://doi. org/10.1016/j.quascirev.2017.02.016

Hartemink, A.E., Bockheim, J.G., 2013, Soil genesis and classification: Catena, 104, 251-256. https://doi.org/10.1016/j. catena.2012.12.001

Hernández-Jiménez, A., Vera-Macías, L., NavedaBasurto, G.A., Guzmán-Cedeño, A.M., VivarArrieta, M., Zambrano, T.R., Mesías-Gallo, F., Ormanza, K., León-Aguilar, R.V., LópezAlava, G.A., 2017, Variaciones en algunas propiedades del suelo por el cambio de uso de la tierra en las partes media y baja de la microcuenca membrillo, Manabí, Ecuador: Cultivos Tropicales, 38, 50-56.

Instituto Mexicano de Tecnología del Agua (IMTA), 1989, Manual de clasificación, cartografía e interpretación de suelos con base en el sistema de texonomía de suelos: Cuernavaca, Instituto Mexicano de Tecnología del Agua, 297 p.

Instituto Nacional de Estadística y Geografia (INEGI), 2011a, Ortofotomapas (en línea): Marco Geoestadístico E15d11f, E15d1le, E15d21a, E15d21b, E15d21c, E15d21d, E15d21e1 y E15d21f, escala 1:50000, disponible en <https://www.inegi.org.mx/temas/mg/ default.html\#Descargas $>$, consultado 15 de noviembre de 2016.

Instituto Nacional de Estadística y Geografia (INEGI), 2011b, Modelo digital de elevación, escala 1:50000: San Luis Potosí, disponible en <http:// www.inegi.org.mx/geo/contenidos/datosrelieve/ continental//Descargas.asx $>$, consultado el $30 \mathrm{de}$ Junio de 2013.

Instituto Nacional de Estadística y Geografia (INEGI), 2016, Anuario estadístico y geográfico de Tabasco, 2016: México, Instituto Nacional de Estadística y Geografia (INEGI), 461 p.

IUSS Working Group (WRB), 2015, World Reference Base for Soil Resources 2014, update 2015, International soil classification system for naming soils and creating legends for soil maps: World Soil Resources Reports, n. 106: Rome, $192 \mathrm{p}$. 
Kemp, J., Pietsch, T., Gonzt, A., Olley, J., 2017, Lacustrine-fluvial interactions in Australia's Riverine Plains: Quaternary Science Reviews, 166, 1-11. https://doi.org/10.1016/j. quascirev.2017.02.015

Lanza, E.G. de la, Ortiz-Pérez, M.A., Carbajal-Pérez J.L., 2013, Diferenciación hidrogeomorfológica de los ambientes costeros del Pacífico, del Golfo de México y del mar Caribe: Investigaciones Geográficas, 81, 33-50. https://doi. org/10.14350/rig.33375

López-Castañeda, A., Palma-López, D.J., ZavalaCruz, J., Ortiz-Solorio, G.A., Tinal-Ortiz, S., Valdez-Lazalde, J.R., 2017, Cartografía de los suelos en la región de Los Ríos, Tabasco, México: uso del modelo digital de elevación: Agroproductividad, 10, 22-28.

Martín-Vide,J.P., 2015, Restauración del Río Besòs en Barcelona. Historia y lecciones aprendidas: Revista Iberoamericana del Agua, 2(1), 51-60. https://doi.org/10.1016/j.riba.2015.07.001

Martínez-Villegas, N., 2007, An overview of different soil classification systems used in Mexico:Terra Latinoamericana, 25(4), 357-362.

Medina-Orozco, L.E., García-Calderón, N.E., García-Oliva, F., Ikkone, E., 2014, Suelos de Humedales del lago de Pátzcuaro, Michoacán, México: Tecnología y Ciencias del Agua, 5, 111-124.

Miller, B.A., Schaetzl, R.J., 2014, The historical role of base maps in soil geography: Geoderma, 230-231, 329-339. https://doi.org/10.1016/j. geoderma.2014.04.020

Moreno-Cáliz, E., Gutiérrez-Castorena, M. del C., Guerrero-Peña, A., Ortiz-Solorio, C.A., Palma-López, D.J., 2004, Micromorfología en la clasificación de Histosoles en humedales de Tabasco, México: Terra Latinoamericana, 22, 153-160.

Norma Oficial Mexicana (NOM-021-REGNAT), 2000, Establece las especificaciones de fertilidad, salinidad y clasificación de suelos, estudio, muestreo y análisis. Diario oficial de la federación: México, Semarnat 85 p.
Ortiz-Pérez, M.A., Siebe, C., Cram, S., 2005, Diferenciación ecogeográfica de Tabasco, en Bueno, J., Álvarez, F., Santiago, S. (eds.), Biodiversidad del Estado de Tabasco: México, Conabio e Instituto de Biología de la UNAM, 305-322.

Paladino, I.R., Irigoin, J., Moretti, L., Civeira, G., 2017, Relaciones Geopedológicas y análisis multivariado de los atributos edáficos asociados a las dunas longitudinales del noroeste de la provincia de Buenos Aires, Argentina: Revista de la Asociación Geológica Argentina, 74, 373-383.

Palma-López, D.J., Cisneros, D.J., 2000, Plan de uso sustentable de los suelos de Tabasco: Villahermosa, Fundación Produce Tabasco, vol. I, $115 \mathrm{p}$.

Palma-López, D.J., Cisneros, D.J., Moreno, C.E., Rincón-Ramírez, J.A., 2007, Suelos de Tabasco: su uso y manejo sustentable: Villahermosa, Colegio Postgraduados ISPROTAB-FUPROTAB, $184 \mathrm{p}$.

Palma-López, D.J., Zavala, C.J., Bautista, Z.F., Morales, G.M.A., López, C.A., Shirma, T.E.D., Gallegos, T.J., 2012, Estudio sobre el plan de uso sustentable de los suelos del estado de Campeche: San Francisco de Campeche, Colegio de Postgraduados,Secretaría de Desarrollo Rural de Campeche, reporte técnico, $141 \mathrm{p}$.

Palma-López, D.J., Zavala-Cruz, J., BautistaZuñiga, F., Morales-Garduza, M.A., López-Castañeda, A., Shirma-Torres, E.D., Sánchez-Hernández, R., Peña-Peña, A., Tinal-Ortiz, S., 2017, Clasificación y cartografía de suelos del estado de Campeche, México: Agroproductividad, 10, 71-78.

Porta, J., López-Acevedo, M., Poch, R.M., 2013, Edafología. Uso y Protección de suelos: Madrid, España, Mundi-Prensa, 608 p.

Ramos-Reyes, R., Zavala-Cruz, J., GamaCampillo, L.M., Pech-Pool, D., Ortiz-Pérez, M.A., 2016, Indicadores geomorfológicos para evaluar lavulnerabilidad por inundación 
ante el ascenso del nivel del mar debido al cambio climático en la costa de Tabasco y Campeche, México: Boletín de la Sociedad Geológica Mexicana, 68(3), 581-598. https:// doi.org/10.18268/bsgm2016v68n3a12

Romo, M. de L., Ortiz-Pérez, M.A., 2001, Riesgo de inundación en la llanura fluvial del curso bajo del río San Pedro, Nayarit: Investigaciones Geográficas, 45, 7-23. https://doi.org/10.14350/rig.59142

Salgado-García, S., Palma-López, D.J., LagunesEspinoza, L.C., Ortiz-García, C.F., AscencioRivera, J.M., 2005, Bases para generar un programa sustentable de fertilización en un ingenio de Tabasco, México: Interciencia, 30, 395-403.

Salgado-García, S., Palma-López, D.J., ZavalaCruz, J., Lagunez-Espinoza, L.C., CórdovaSánchez, S., Castelán-Estrada, M., Ortiz-García, C.F., Rincón-Ramírez, J.A., 2015, Sistema integrado para recomendar dosis de fertilizantes en Palma de aceite (SIRDF): Región Ríos de Tabasco: Cárdenas, Tabasco, Grupo SIRDF PA-LPi: AESS, Colegio de Postgraduados, Campus Tabasco, $118 \mathrm{p}$.

Servicio Geológico Mexicano (SGM), 2008, Carta geológico-minera: Estados de Chiapas y Tabasco, escala 1: 500 000: Pachuca, México.

Soil Survey Staff, 2014, Soil taxonomy: A basic system of soil classification for making and interpreting soil surveys: Natural Resources Conservation Service, U.S. Department of Agriculture, 399 p.

Solís-Castillo, B., Ortiz-Pérez, M.A., SolleiroRebolledo, E., 2014, Unidades geomorfológicoambientales de las tierras bajas mayas de Tabasco-Chiapas en el río Usumacinta: un registro de los procesos aluviales y pedológicos durante el Cuaternario: Boletín de la Sociedad Geológica Mexicana, 66(2), 279-290. https:// doi.org/10.18268/bsgm2014v66n2a5

Zavala-Cruz, J., Castillo, A.O., Ortiz, C.A.I., PalmaLópez, D.J., Juárez, L.J.F., Salgado-García, S., Rincón-Ramírez, J.A., Moreno, C.E., Ramos, R.R., 2009, Capacidad de uso del suelo urbano en Tabasco, con base en el suelo, uso actual y vegetación: Tabasco, Colegio de Postgraduados, Secretaría de Asentamientos y Obras Públicas, Secretaría de Recursos Naturales y Protección Ambiental, 204 p.

Zavala-Cruz, J., Palma-López, D.J., Fernández, C.C.R., López, C, A., Shirman, T.E., 2011, Degradación y conservación de suelos en la cuenca del Río Grijalva, Tabasco: Villahermosa, Tabasco, Colegio de Postgraduados, 90 p.

Zavala-Cruz,J., Palma-López, D.J., Morales, G.M.A., 2012, Clasificación de suelos de la cuenca baja del río Tonala, Tabasco, en Zavala, C.J.y García, L.E. (eds.), Suelo y vegetación de la cuenca baja del Río Tonalá, Tabasco: Cárdenas, Tabasco, Colegio de Postgraduados, $142 \mathrm{p}$.

Zavala-Cruz, J., Salgado-García, S., Marín-Aguilar, A., Palma-López, D.J., Castelán-Estrada, M., Ramos, R.R., 2014, Transecto de suelos en terrazas con plantaciones de cítricos en Tabasco: Ecosistemas y recursos agropecuarios, 1, 123-137.

Zavala-Cruz, J., Jiménez-Ramírez, R., PalmaLópez, D.J., Bautista-Zuñiga, F., Gavi-Reyes, F., 2016a, Paisajes geomorfológicos: base para el levantamiento de suelos en Tabasco, México: Ecosistemas y Recursos Agropecuarios, 3, 161-171.

Zavala-Cruz, J., Morales-Garduza, M.A., VargasVillamil, L.M., Palma-López, D.J., OrtízSolorio, C.A., 2016b, Capacidad de uso del suelo urbano en planicies fluviales costeras: El caso de Villahermosa, Tabasco, México: Interciencia, 41, 296-304.

Zinck, J.A., 1989, Physiography and soil. Lecture notes for soil students. Soil Science Division, Soil survey courses subject matter: Enschede, The Netherlands, ITC, $161 \mathrm{p}$.

Zinck, A.J., 2012, Geopedología: elementos de geomorfología para estudios de suelos y de riesgos naturales: Enschede, The Netherlands, Faculty of Geo-Information Science and Earth Observation, 123 p. 